\title{
Pointwise Gradient Estimates of Solutions to Onedimensional Nonlinear Parabolic Equations
}

\author{
Philippe BENILAN $(\dagger)^{*}$ and Jesús Ildefonso DÍAZ ${ }^{\dagger}$
}

June 7, 2002

\begin{abstract}
We present here an improved version of the method introduced by the first author to derive pontwise gradient estimates for the solutions of one-dimensional parabolic problems. After considering a general qualinear equation in divergence form we apply the method to the case of a nonlinear diffusion-convection equation. The conclusion are stated first for classical solutions and then for generalized and mil solutions. In the case of unbounded initial datum we obtain several regularizing effects for $t>0$. Some unilateral pointwise gradient estimates are also obtained. The case of the Dirichlet problem is also considered. Finally, we collect, in the last section, several comments showing the connections among these estimates and the study of the free boundaries associated to the solutions of the diffusion-convection equation.

Key words: Pointwise gradient estimates, one-dimensional parabolic equations, non linear diffusion-convection equation, regularizing effects, unilateral estimates, interfaces.

200 Mathematical Subject Classification: 35K65, 35K10, 35R35, 76D27.
\end{abstract}

\section{Introduction}

This paper deals with a very old task which seems to have their origins in the work by S.N. Bernstein on the beginnings of the last century ([10]): find a priori pointwise estimates on the (spatial) gradient of solutions of nonlinear second order equations. To be more precise we shall consider onedimensional parabolic equations.

Our main purpose is to present here an improved version of a general method introduced, originally, by the first author (see Section I. 2 of Benilán [6]). The method has important differences with respect the way in which D.G. Aronson [3] derived, in 1969, this type of estimates for the porous media equation. His technique was later applied by different authors to many other nonlinear degenerate equations (see references in the surveys [31] and [41]). Such a already classical technique consists of, firstly, introducing the variable $u=f(w)$ with a concave function of the form $f(r)=C_{1} r\left(C_{2}-r\right)$, for some suitable constants $C_{i}>0$ and, secondly, estimating the value of $\left|v_{x}\right|$ at a maximum of the auxiliary function $z=\zeta^{2} w_{x}^{2}$, with $\zeta$ a cutoff function.

The method here presented seems to be better adapted to recognize the different nonlinear terms involved in the equation and so, it allows to obtain sharp pointwise gradient estimates. Moreover, our method provides quantitative estimates. In many cases these estimates have an important physical meaning and allow to deduce many other qualitative properties of the solutions.

We shall limit the exposition to a general class of equations in divergence form

$$
u_{t}=\sigma\left(u, u_{x}\right)_{x}
$$

${ }^{*}$ Équipe de Mathematiques, UA CNRS 741, Université de Franche-Comté, 25030 Besançon, France.

${ }^{\dagger}$ Facultad de Matemáticas, Universidad Complutense de Madrid, 20040 Madrid, Spain. 
but the method also applies to more general equations (see the exposition made in [6]). In order to explain some additional details, we consider the special case of the nonlinear diffusion-convection equation

$$
u_{t}=\varphi(u)_{x x}+\psi(u)_{x}
$$

Equation (2) appears in many different contexts. For instance, unsaturated soil-moisture flows are modelled by $(2)$ under the condition $\varphi^{\prime}(0)=0$. In that case the equation becomes degenerate and due to that a very rich structure arise concerning many different aspects: finite speed of propagation, stability results, etc. In the context of gas flow in a porous medium, the expression

$$
V(t, x)=-\frac{\varphi(u(x, t))_{x}+\psi(u(x, t))}{u(x, t)}
$$

represents the Eulerian description of the velocity at the position $x$ and the time $t$ of the "homogenized gas" (see, [18], [14]). It seems natural to expect boundedness of $V$ which, in fact, leads to "optimal gradient bounds" for the solution $u$ of (2) in some special cases as, for instance,. $\varphi(u)=u^{m}, \psi(u)=u^{n}, 1<m, 1 \leq n$. In that case, it is possible to find out some special solutions for which the modulus of continuity found via this gradient estimate can not be improved (see, e.g., [26], [21] and [29]).

The nature of "optimal gradient bounds" is much less evident in other contexts in which equation (2) arises (as, for instance, in Plasma Physics where $\varphi(u)=u^{m}$ and $0<m<1$ [11]) or even in the context of equation (2) when convection dominates over diffusion: $\varphi(u)=u^{m}, \psi(u)=$ $u^{n}$ and $\left.0<n \leq 1<m:[16]\right)$.

One of the main features of the method, as presented here, is that it endows us with a "simple" analytical argument that allows to find sharp gradient bounds independently on the physical arguments involved in the concrete formulation. Another characteristic of the method is that it can be used locally in time and space and, in particular, it illustrates some regularizing effects (for instance some gradient bounds take place for any $t>0$, independently either it already holds at $t=0$ or not).

The main idea of the method is to find an auxiliary nonlinear parabolic operator $\mathcal{L}$ in such a way that a pointwise gradient estimate of the type

$$
\sigma\left(u, u_{x}\right) \leq k(t, x, u)
$$

holds as a consequence of the comparison principle applied to a function of the form $p=\sigma\left(u, u_{x}\right) / k(., ., u)$, in the kernel of operator $\mathcal{L}$, and a constant function, i.e.

$$
\mathcal{L}(p)=0 \leq \mathcal{L}(1)
$$

In this way, the possible choices of function $k(t, x, u)$ are determined by the condition $\mathcal{L}(1) \geq 0$.

The contains of the paper is the following: Section 2 is devoted to a general presentation of the method for equations (1) and its particularization (2) in the context of classical solutions. The application to the case of weaker notions of solution is carried out in Section 3. Pointwise gradient estimates are first obtained for the generalized solution of the Cauchy problem associated to (2) in Subsection 3.1. An unidirectional pointwise gradient estimate, that cease to be true if the direction of the spatial variable $x$ is changed, is obtained in Subsection 3.2. The case of unbounded initial data and the proof of regularizing effects in the class of mild solutions are developed in Subsection 3.3. The derivation of pointwise gradient estimates for the case of a finite spatial interval is considered in Subsection 3.4. Finally, some comments on the connection between pointwise gradient estimates and the study of the free boundaries defined as the boundaries of the support of the solution are collected in Subsection 3.5. 


\section{The general method}

In this section we introduce the main idea of the method. As usual, pointwise gradient estimates will be obtained as a priori estimates for any possible solution $u$. So, at the first step, they will be obtained in the more favorable setting of smooth solutions. Since the resulting estimates will be independent of the regularity used in the proof, they can be extended to the framework of weaker solutions. This second step will be illustrated in the next section.

\subsection{A general quasilinear equation}

Let $Q$ be an open regular set of $\mathbb{R} \times \mathbb{R}$ and let $u$ be a classical solution of the equation

$$
u_{t}=\sigma\left(u, u_{x}\right)_{x} \quad \text { in } Q
$$

We assume $u \in C^{2,3}(Q)$ such that

$$
m_{-} \leq u(t, x) \leq m_{+} \text {on } Q
$$

for some $m_{-} \leq m+$. We also assume that $\sigma \in C^{2}\left(\left[m_{-}, m_{+}\right] \times \mathbb{R}\right)$.

Our main goal is to obtain pointwise estimates as (3) for suitable auxiliary functions $k$. As mentioned before, we start by assuming the regularity conditions: $k \in C^{2}\left(Q \times\left[m_{-}, m_{+}\right]\right)$and $k \neq$ 0 . The principal ingredient of the method is to find a (possibly nonlinear) parabolic operator, $\mathcal{L}$, vanishing when applied to the function

$$
p(t, x):=\frac{\sigma\left(u(t, x), u_{x}(t, x)\right)}{k(t, x, u)} \quad(t, x) \in Q
$$

(some other choices of function $p$ are also of interest: see [6]).

Using (5) we find

$$
u_{t}=(k p)_{x}=\left(k_{x}+k_{u}+k_{u} u_{x}\right) p+k p_{x}
$$

and differentiating (8) with respect to $x$,

$$
u_{t x}=\left(k_{x x}+2 k_{x u} u_{x}+k_{u u} u_{x}^{2}+k_{u} u_{x x}\right) p+2\left(k_{x}+k_{u} u_{x}\right) p_{x}+k p_{x x}=\sigma_{u} u_{x}+\sigma_{u_{x}} u_{x x} .
$$

Differentiating (7) with respect to $t$ we have

$$
p_{t} k+p\left(k_{t}+k_{u} u_{t}\right)=\sigma_{u} u_{t}+\sigma_{u_{x}} u_{t x} .
$$

Multiplying (8) by $\sigma_{u}$ and (9) by $\sigma_{u_{x}}$ and adding the resulting expressions we find that

$$
\widetilde{\mathcal{L}}\left(p, u_{x}\right)=0
$$

where

$$
\begin{aligned}
\widetilde{\mathcal{L}}\left(p, u_{x}\right)= & p_{t} k+p k_{t}+\left(p k_{u}-\sigma_{u}\right)\left\{\left(k_{x}+k_{u} u_{x}\right) p+k p_{x}\right\} \\
& -\sigma_{u_{x}}\left[\left(k_{x x}+2 k_{x u} u_{x}+k_{u u} u_{x}^{2}\right) p+2\left(k_{x}+k_{u} u_{x}\right) p_{x}+k p_{x x}\right] \\
& -k_{u} p\left[p_{x} k+p\left(k_{x}+k_{u} u_{x}-\sigma_{u} u_{x}\right] .\right.
\end{aligned}
$$

In order to obtain an expression independent of $u_{x}$ we assume

$$
\sigma_{u_{x}}>0 \text {. }
$$

Thus we can define $S(u, \cdot):=\sigma(u, \cdot)^{-1}$ and so $u_{x}=S(u, k p)$. Finally, in order to find an operator $\mathcal{L}$ such that $\mathcal{L}(p)=0$ we define $\mathcal{L}$ by 


$$
\mathcal{L}(p):=\widetilde{\mathcal{L}}(p, S(u, k p))
$$

(the dependence on $u$ of the right hand side is understood as a $(t, x)$-dependent coefficient). The exact form of $\mathcal{L}(p)$ is irrelevant for our purposes. Our main aim is to compare $p$ with 1 and so the relevant expression is $\mathcal{L}(1)$, i.e.

$$
\mathcal{L}(1)=k_{t}+\left(k_{u}-\sigma_{u}(\widetilde{S}(k)) k_{x}-\sigma_{u_{x}}(\widetilde{S}(k))\left\{k_{x x}+2 k_{x u} \widetilde{S}(k)+k_{u u} \widetilde{S}(k)^{2}\right\}\right.
$$

where we used the notation $\sigma_{u}(\widetilde{S}(k))=\sigma_{u}(u, \widetilde{S}(k))$ and $\widetilde{S}(k):=S(u, k)$.

The following result is a trivial consequence of the maximum principle (see, e.g., the presentation made in .[5]):

Lemma 1. Assume (10),

$$
\begin{gathered}
k(t, x, u)>0 \quad \text { on } Q \times\left(m_{-}, m_{+}\right), \\
\mathcal{L}(1) \geq 0 \quad \text { in } Q \\
\sigma\left(u, u_{x}\right)(t, x) \leq k(t, x, u(t, x)), \quad(t, x) \in \partial_{p} Q,
\end{gathered}
$$

where $\partial_{p} Q$ denotes the parabolic boundary of $Q$. Then

$$
\sigma\left(u, u_{x}\right)(t, x) \leq k(t, x, u(t, x)) \text { for any }(t, x) \in \bar{Q}
$$

A concrete boundary value problem for which it is easy to check assumption (14) corresponds to the case of Neumann type boundary conditions.

Lemma 2. Let $I=(a, b)$ with $-\infty \leq a<b \leq+\infty$ and let $Q=(0, T) \times I$ where $0<T \leq+\infty$. Let $u$ be a classical solution of (5) on $Q$. Assume (10), (12), (13),

$$
\begin{gathered}
\sigma\left(u, u_{x}\right)(0, x) \leq k(u)(0, x) \quad \text { for all } x \in I, \\
\left\{\begin{array}{l}
\left(u_{t}-k_{x}-k_{u} u_{x}\right)(t, a) \geq 0 \text { if } a>-\infty, \text { for all } t \in(0, T), \\
\left(u_{t}-k_{x}-k_{u} u_{x}\right)(t, b) \leq 0 \text { if } b<+\infty, \quad \text { for all } t \in(0, T),
\end{array}\right.
\end{gathered}
$$

and

$$
\left\|\frac{k_{x}+k_{u} u_{x}}{k}\right\|_{L^{\infty}((0, T) \times \partial I)}<\infty .
$$

Then estimate (15) holds.

Proof. From (8) we have that

$$
-p_{x}+p\left[-\left\{\frac{k_{x}+k_{u} u_{x}}{k}\right\}\right]=\frac{-u_{t}}{k}
$$

So, if $\nu$ denotes the exterior "unit outer normal" to $\partial I$ (i.e. $v(a)=-1, v(b)=1$ ), we deduce that

$$
-p_{x} v+p\left[-v\left\{\frac{k_{x}+k_{u} u_{x}}{k}\right\}\right]=-\frac{u_{t} v}{k} \text { on }(0, T) \times \partial I .
$$

Since we assume (18) we can apply the comparison principle. Taking $\bar{p}(t, x)=1$, assumption (16) implies that $p \leq \bar{p}$ on $\{0\} \times(a, b)$ and (17) implies that

$$
-\bar{p}_{x} v+\bar{p}\left[-v\left\{\frac{k_{x}+k_{u} u_{x}}{k}\right\}\right] \leq-\frac{u_{t} v}{k} \text { on }(0, T) \times \partial I .
$$


In consequence $p \leq \bar{p}$ on $[0, T] \times \bar{I}$.

In the special case of

$$
k(t, x, u)=\theta(u)
$$

we deduce, from (11), that

$$
\mathcal{L}(1)=-\sigma_{u_{x}}(\widetilde{S}(\theta)) \widetilde{S}(\theta)^{2} \theta^{\prime \prime}
$$

and then the following result holds:

Corollary 1. Let $u$ be a smooth solution of (5) satisfying (6). Assume (10) and let $\theta \in C^{2}\left(\left[m_{-}, m_{+}\right]\right)$ such that

$$
\theta>0 \text { and } \theta^{\prime \prime} \leq 0 \text { on }\left[m_{-}, m_{+}\right]
$$

Then

$$
\frac{\sigma\left(u, u_{x}\right)}{\theta(u)} \leq \sup _{\partial_{p} Q} \frac{\sigma\left(u, u_{x}\right)}{\theta(u)}
$$

Remark 1. Conclusion (22) expresses a "conservation of the regularity": if the estimate is true on the parabolic boundary of $Q$ it also holds in the interior of $Q$. We emphasize the large generality on $\theta$ assumed in (21) and that, by passing to the limit, it is possible to extend the conclusion for $\theta$ concave such that $\theta \geq 0$. As a general remark, we point out that the Bernstein technique, as described before, still works in several space dimensions for radially symmetric solutions (see, for instance, [24], for a related result). $(2)$.

In the rest of this section we illustrate the applicability of the method by considering equation

\subsection{A nonlinear diffusion-convection equation}

As it was already mentioned, a physically relevant special case of equation (1) is

$$
u_{t}=\varphi(u)_{x x}+\psi(u)_{x} \text { in } Q
$$

where $\varphi$ is a nondecreasing function with $\varphi(0)=0$. For the sake of simplicity, we assume in this subsection that $\varphi \in C^{2}\left(\left[m_{-}, m_{+}\right]\right), \varphi^{\prime}>0$ and $\psi \in C^{1}\left(\left[m_{-}, m_{+}\right]\right)$. In the applications we shall present here, we assume the $(t, x)$-dependence of function $k$ of the form

$$
k(t, x, u)=\rho(t, x) \theta(u) .
$$

Then, Lemma 1 leads to the following result

Lemma 2. Let $Q \subset \mathbb{R}^{+} \times \mathbb{R}$. Let $u$ be a smooth solution of (23) satisfying (6). Let $\rho>0$ and $\theta>0$ be smooth functions such that

$$
\begin{gathered}
0 \leq \frac{\rho_{t}}{\rho}+\left[\theta^{\prime}-\varphi^{\prime \prime}\left(\frac{\rho \theta-\psi}{\varphi^{\prime}}\right)-\psi^{\prime \prime}\right] \frac{\rho_{x}}{\rho} \\
-\varphi^{\prime}\left[\frac{\rho_{x x}}{\rho}+2 \frac{\rho_{x}}{\rho} \frac{\theta^{\prime}}{\theta}\left(\frac{\rho \theta-\psi}{\varphi^{\prime}}\right)+\frac{\theta^{\prime \prime}}{\theta}\left(\frac{\rho \theta-\psi}{\varphi^{\prime}}\right)^{2}\right] \quad \text { in } Q \\
\varphi(u)_{x}+\psi(u) \leq \rho \theta(u) \quad \text { on } \partial_{\rho} Q .
\end{gathered}
$$

Then

$$
\varphi(u)_{x}+\psi(u) \leq \rho \theta(u) \quad \text { on } Q
$$


In the next section we shall give several applications of Lemma 2 to the case of weak solutions of (23). Nevertheless, some previous consequences for the cases in which condition (26) is trivially satisfied (the Cauchy and the Neumann problems) can be easily derived for the special choice $\rho(t, x)=1 / \sqrt{t}$.

Corollary 2. A. Let $Q=(0, T) \times \mathbb{R}$ with $0<T \leq \infty$ and $\theta \in C^{2}\left(\left[m_{-}, m_{+}\right]\right)$satisfying $\theta>0$ and

$$
\frac{\varphi^{\prime}}{2}+\theta \theta^{\prime \prime}\left(1-\psi \frac{\sqrt{t}}{\theta}\right)^{2} \leq 0 \quad \text { for any } t \in(0, T] \quad \text { on }\left[m_{-}, m_{+}\right] .
$$

Then, if $u$ is a smooth solution of (23) on $Q$ satisfying (6), we have the estimate

$$
\varphi(u)_{x}+\psi(u) \leq \frac{\theta(u)}{\sqrt{t}} \text { on }(0, T] \times \mathbb{R} .
$$

B. Let $I=(a, b)$ with $-\infty<a<b<+\infty$ and $Q=(0, T) \times I$. Assume that $\theta$ satisfies the conditions of part $\mathrm{A}$ and let $u$ be a smooth solution of equation (23) on $Q$ verifying (6) and the homogeneous Neumann boundary conditions

$$
0=\left(\varphi(u)_{x}+\psi(u)\right)(t, a)=\left(\varphi(u)_{x}+\psi(u)\right)(t, b) \text { for } t \in(0, T) .
$$

Then inequality $(29)$ holds on $(0, T] \times I$. The same conclusion holds if we replace the boundary condition (30) by

$$
0=\varphi(u)_{x}(t, a)=\varphi(u)_{x}(t, b)
$$

and assume that $\psi \leq 0$ on $\left(m_{-}, m_{+}\right)$

Remark 2. In contrast to Corollary 1 the above result shows the first "regularizing effect": estimate (29) holds for any $t>0$, independently of either it takes place at $t=0$ or not. Other regularizing effects will be given in Subsection 3.3.

Another interesting choice of function $\rho(t, x)$ leading to local effects, independently of the boundary conditions, is $\rho(t, x)=\xi(x) / \sqrt{t}$ with $\xi(x)$ vanishing on the boundary of the spatial domain.

Corollary 3. Let $I=(a, b)$ with $-\infty<a<b \leq+\infty$ and $Q=(0, T) \times I$. Assume that $\theta \in C^{2}\left(\left[m_{-}, m_{+}\right]\right), \theta>0, \xi \in C^{2}(I), \xi>0$ on $I$ and $\xi=0$ on $\partial I$. Assume that

$$
\begin{aligned}
0 \leq & -\frac{1}{2 t}+\left[\theta^{\prime}-\varphi^{\prime \prime}\left(\frac{\theta \xi / \sqrt{t}-\psi}{\varphi^{\prime}}\right)-\psi^{\prime \prime}\right] \frac{\xi_{x}}{\xi} \\
& -\varphi^{\prime}\left[\frac{\xi_{x x}}{\xi}+2 \frac{\xi_{x}}{\xi} \frac{\theta^{\prime}}{\theta}\left(\frac{\theta \xi / \sqrt{t}-\psi}{\varphi^{\prime}}\right)+\frac{\theta^{\prime \prime}}{\theta}\left(\frac{\theta \xi / \sqrt{t}-\psi}{\varphi^{\prime}}\right)^{2}\right]
\end{aligned}
$$

Let $u$ be a smooth solution of equation (23) on $Q$ satisfying (6). Then we have the inequality

$$
\varphi(u)_{x}+\psi(u) \leq \frac{\theta(u) \xi(x)}{\sqrt{t}} \text { on }(0, T] \times I .
$$

Condition (32) leads to interesting results once we know more about the functions $\varphi$ and $\psi$, for instance, if $\varphi(u)=u^{m}, \psi(u)=u^{n}$ for some positive $m$ and $n$ (see Subsection 3.4). 


\section{Applications to generalized and mild solutions}

Corollaries 2 and 3 will be used in this section for two different objectives: (i) to extend estimates (29) and (33) to weaker notions of solution of the Cauchy and Dirichlet problems associated to equation (23) (and so under much more generality on the data $\varphi, \psi$ and $u_{0}$ ), and, (ii) to present explicitly different choices of the function $\theta$ according $\varphi$ and $\psi$.

\subsection{Generalized solutions}

We start by considering the class of nonnegative bounded generalized solutions of the Cauchy problem

$$
\begin{gathered}
0 \leq u_{0}(x) \leq M_{0} \text { for } \text { any } x \in \mathbb{R} . \\
\begin{cases}u_{t}=\varphi(u)_{x x}+\psi(u)_{x} & \text { in }(0, T) \times \mathbb{R}, \\
u(0, x)=u_{0}(x) & x \in \mathbb{R}\end{cases}
\end{gathered}
$$

with

$$
0 \leq u_{0}(x) \leq M_{0} \text { for } x \in \mathbb{R}
$$

for some $M_{0}$. We assume that

$$
\varphi, \psi \in C^{0}\left(\left[0, M_{0}\right]\right), \varphi(0)=0, \varphi \text { strictly increasing }
$$

as well as the following technical conditions (see Subsection 3.3)

$$
\left\{\begin{array}{l}
\exists \delta_{0}>0 \text { such that } \varphi, \psi \in C^{2}\left(\left(0, M_{0}+\delta_{0}\right]\right) \text { and } \\
\varphi^{\prime \prime} \text { and } \psi^{\prime \prime} \text { are locally Hölder continuous on }\left(0, M_{0}+\delta_{0}\right] .
\end{array}\right.
$$

We recall that given $0<T \leq+\infty$, a function $u(t, x)$ is said to be a nonnegative bounded generalized solution of equation $(23)$ if $u \in C([0, T] \times \mathbb{R})$,

$$
0 \leq u(t, x) \leq M_{0} \text { for. } x \in \mathbb{R} \text { and } t \in[0, T]
$$

for some $m>0$ and satisfies the integral identity

$$
\begin{aligned}
\int_{t_{1}}^{t_{2}} \int_{x_{1}}^{x_{2}}\left\{u \phi_{t}+\varphi(u) \phi_{x x}-\psi(u) \phi_{x}\right\} d x d t= & \int_{x_{1}}^{x_{2}}\left\{u\left(t_{2}, x\right) \phi\left(t_{2}, x\right)-u\left(t_{1}, x\right) \phi\left(t_{1}, x\right)\right\} d x \\
& +\int_{t_{1}}^{x_{t 2}}\left\{u\left(t, x_{2}\right) \phi\left(t, x_{2}\right)-u\left(t, x_{1}\right) \phi\left(t, x_{1}\right)\right\} d t
\end{aligned}
$$

for all non-empty bounded rectangles $\left(t_{1}, t_{2}\right) \times\left(x_{1}, x_{2}\right)$ and nonnegative functions $\phi \in C^{1,2}\left(\left[t_{1}, t_{2}\right] \times\right.$ $\left.\left[x_{1}, x_{2}\right]\right)$ such that $\phi\left(t, x_{1}\right)=\phi\left(t, x_{2}\right)=0$ for all $t \in\left[t_{1}, t_{2}\right]$.

We recall (see, e.g., [16]) that any generalized solution is a weak solution in the sense that the above integral identity can be replaced by the condition $u_{t}=\varphi(u)_{x x}+\psi(u)_{x}$ in $D^{\prime}(Q)$.

Different results on the existence and uniqueness of a generalized solution $u$ of the Cauchy Problem (35) are today available in the literature (see e.g. [9] [20] and [13]). For our present purposes we shall made use of the results of Gilding [23] requiring, merely, the continuity of the initial datum. We have:

Theorem 1. Assume that $\varphi$ and $\psi$ satisfy (37) and (38). Let $u_{0} \in C^{0}(\mathbb{R})$ satisfying (36). Let $u \in C([0, T] \times \mathbb{R})$ be the generalized solution to the Cauchy problem (35). Assume that there exists a function $\lambda \in C^{0}\left(\left[0, M_{0}\right]\right)$, concave, increasing and such that

$$
M<\infty
$$

where 


$$
M:=\int_{0}^{M_{0}} \frac{d \varphi(s)}{\lambda(s) \lambda^{\prime}(s)} .
$$

Finally, assume that there exists a constant $C \geq 0$ such that

$$
\psi(s) \leq C \lambda(s) \text { for any } s \in\left(0, M_{0}\right) .
$$

Then

$$
\varphi(u)_{x}+\psi(u) \leq\left[\sqrt{4 C^{2} T+2 M}+2 C \sqrt{T}\right] \frac{\lambda(u)}{\sqrt{t}} \text { in } D^{\prime}((0, T) \times \mathbb{R}) .
$$

Before giving the proof we shall state two immediate consequences.

Corollary 3. Let $\varphi$ and $\psi$ satisfying (37) and (38). Assume $\psi$ be such that

$$
\psi(s) \leq C \sqrt{s} \text { for any } s \in\left(0, M_{0}\right),
$$

for some constant $C \geq 0$. Then, if $u_{0} \in C^{0}(\mathbb{R})$ satisfies (36) and $u$ is the generalized solution to the Cauchy Problem (35) we have the estimate

$$
\varphi(u)_{x}+\psi(u) \leq 2 \sqrt{\frac{u \varphi\left(M_{0}\right)}{t}} \text { in } D^{\prime}((0, T) \times \mathbb{R}) .
$$

Corollary 4. Let $\varphi$ and $\psi$ satisfying (37) and (38). Let $u_{0} \in C^{0}(\mathbb{R})$ satisfying $(36)$ and $u$ be the generalized solution of the Cauchy Problem (35). Then $\varphi(u(t, \cdot))_{x} \in L^{\infty}(\mathbb{R})$ and

$$
\left\|\varphi(u(t, \cdot))_{x}\right\|_{L^{\infty}(\mathbb{R})} \leq 2\left[\frac{\varphi\left(M_{0}\right) M_{0}}{2}\right]^{1 / 2}+N
$$

for any $t>0$, where

$$
N=\sup _{\left[0, M_{0}\right]} \psi-\inf _{\left[0, M_{0}\right]} \psi \cdot \square
$$

Proof of Corollaries 3 and 4. Corollary 3 follows from the fact that function $\lambda(s)=\sqrt{s}$ satisfies (40) for any continuous nondecreasing function $\varphi$. To prove Corollary 4 we point out that $u$ is also a solution of the equation

$$
u_{t}=\varphi(u)_{x x}+\psi_{d}(u)_{x},
$$

where $\psi_{d}(s):=\psi(s)+d$ with $d \in \mathbb{R}$ arbitrary. By choosing $d=-d_{1}$ with $d_{1}=\sup \{\psi(s): s \in$ $\left.\left[0, M_{0}\right]\right\}$ condition (43) is verified for $\psi_{d}$ (since by the maximum principle $u$ we know that $u$ satisfies $\left.0 \leq u \leq M_{0}\right)$. Then (44) implies

$$
\varphi(u)_{x} \leq\left(\frac{2 M_{0} \varphi\left(M_{0}\right)}{t}\right)^{1 / 2}+d_{1}-d_{2}
$$

where $d_{2}=\inf \left\{\psi(s): s \in\left[0, M_{0}\right]\right\}$. To obtain the estimate in the reverse sense we define $\widetilde{u}(t, x)=$ $u(t,-x)$. Then $\widetilde{u}$ satisfies

$$
\widetilde{u}_{t}=\varphi(\widetilde{u})_{x x}+\widetilde{\psi}_{d}(\widetilde{u})_{x}
$$

with $\widetilde{\psi}_{d}(s)=-\psi(s)-d$. Then, by choosing $d=-d_{2}$, we conclude that

$$
-\varphi(u)_{x}=\varphi(\widetilde{u})_{x} \leq 2\left(\frac{M_{0} \varphi\left(M_{0}\right)}{t}\right)^{1 / 2}+d_{1}-d_{2},
$$

and so estimate (45) holds.

Proof of Theorem 1. To get the result we shall perform an approximation process according classical ideas. Our main purpose is to approximate $u$ by a sequence of smooth functions. Let $\delta_{0}$ be given in (38). Fix $\delta \in\left(0, \delta_{0}\right)$ arbitrary and let $k_{0} \in \mathbb{N}$ be such that 


$$
2^{-k_{0}+1} \leq \varphi\left(M_{0}+\delta\right)-\varphi\left(M_{0}\right) .
$$

For $k \geq k_{0}$ we define the cylinder $Q_{k}=(0, T) \times(-k, k)$ and consider the boundary value problem

$$
\begin{gathered}
u_{t}=\varphi(u)_{x x}+\psi(u)_{x} \quad \text { in } Q_{k}, \\
u(t, \pm k)=M_{0} \text { for all } t \in(0, T), \\
u(0, x)=u_{0, k}(x) \text { on }(-k, k),
\end{gathered}
$$

where the sequence $\left\{u_{0, k}\right\}$ satisfies the following properties: (i) $u_{0, k}(x) \leq M_{0}+\delta$ for all $x \in \mathbb{R}$, (ii) there exists $\varepsilon_{k}>0$ such that $\varepsilon_{k} \leq u_{0, k}(x)$ for all $x \in \mathbb{R}$, (iii) there exists $\alpha_{k} \in(0,1]$ such that $u_{0, k} \in C^{2+\alpha_{k}}(\mathbb{R})$, (iv) $u_{0, k+l}(x) \leq u_{0, k}(x)$ for all $x \in \mathbb{R}$, (v) $u_{0, k} \downarrow u_{0}$ as $k \uparrow \infty$ uniformly on compact subsets of $\mathbb{R}$, (vi) $u_{0, k}(x)=M_{0}+\delta$ for all $|x|>k-1 / 2$.

This approximation process was made explicit in [23]. Since properties (ii), (iii) and (vi) holds we can apply the theory of nondegenerate parabolic problems (see e.g. [34]) and so (50), (51), (52) has a unique classical solution $u_{k}(t, x)$. Moreover from (i) and (ii) we deduce that $\epsilon k \leq u_{k}(t, x) \leq M_{0}+\delta$ for all $(t, x) \in Q_{k}$. By the maximum principle, property (iv) implies, that $u_{k+l}(t, x) \leq u_{k}(t, x)$ for all $(t, x) \in \bar{Q}_{k}$ (assumed $\left.k \geq k_{0}\right)$. Hence we can define

$$
u^{*}(x, t)=\lim _{k \nearrow \infty} u_{k}(x, t) \text { for all }(t, x) \in[0, T] \times \mathbb{R} .
$$

It is easy to see that $u^{*}$ is a generalized solution. It is clear that it remains to show that $u^{*} \in$ $C^{0}([0, T] \times \mathbb{R})$ since by the uniqueness of the solution, $u=u^{*}$. The continuity of $u^{*}$ will be obtained similarly to the proof of Theorem 1 of [23]: as in the proof of Corollary 4, we first conclude the gradient estimate for $t \in(0, T]$

$$
\left\|\varphi\left(u_{k}(t, \cdot)\right)_{x}\right\|_{L^{\infty}((-k, k))} \leq 2\left(\frac{\varphi\left(M_{0}+\delta\right)\left(M_{0}+\delta\right)}{t}\right)^{1 / 2}+N
$$

with $N$ given by (46). This implies the existence of a modulus of continuity of $u_{k}$ in $x$ and (by the results of Kruzkov [33] and [20]) also in $t$. This modulus of continuity is independent of $k$ and so, by passing to the limit in $k$, it also applies to $u(t, x)$ for $t \in(0, T]$ and $x \in \mathbb{R}$. The continuity at $t=0$, i.e.the inequalities

$$
u_{0}\left(x_{0}\right) \leq \lim _{(t, x) \longrightarrow\left(0, x_{0}\right)} \inf u(t, x) \leq \lim _{(t, x) \longrightarrow\left(0, x_{0}\right)} \sup u(t, x) \leq u_{0}\left(x_{0}\right)
$$

for any $x_{0} \in \mathbb{R}$, were proved in [20] by using a local barrier function argument which literally applies to our case.

The rest of the proof consists in obtaining the gradient estimate $(42)$, on $(0, T) \times(-k, k)$, for the smooth solutions $u_{k}$ but replacing $M_{0}$ by $M_{\delta}$ in (42), where

$$
M_{\delta}:=\int_{0}^{M_{0}+\delta} \frac{d \varphi}{\lambda(s) \lambda^{\prime}(s)} .
$$

Then, passing to the limit in $k$, we deduce the same estimate for $u$ (but now in the sense of $\left.D^{\prime}((0, T) \times \mathbb{R})\right)$. Finally, as $\delta$ is arbitrary we get $(42)$.

In order to estimate $\varphi\left(u_{k}\right)_{x}+\psi\left(u_{k}\right)$ we apply Lemma 2 with $k(s)=\theta(s) / \sqrt{t}$, where $\theta(s)$ is a convex, increasing and positive function to be determined. Notice that condition (16) trivially holds. On the other hand, from the boundary condition (51) we deduce that $\left(u_{k}(t, \pm k)\right)_{t}=0$ for $t \in(0, T)$. Moreover as $u_{k}(t, x) \leq M_{0}+\delta$ we deduce that $u_{x}(t,-k) \leq 0$ and $u_{x}(t, k) \geq 0$ for all $t$ $\in(0, T)$. Then, conditions (17) holds. Finally, since $u_{k}$ is a positive classical solution, condition (18) is also satisfied. 
Thus, it only remains to check condition (13) or, equivalently, (28). We introduce the function

$$
\theta(s):=e \lambda(s-c j(c))
$$

where $e$ and $c$ are positive constants to be chosen later and $j$ is the convex function defined by

$$
j(s):=\int_{0}^{s} \int_{0}^{r} \frac{d \varphi(z)}{\lambda(z) \lambda^{\prime}(z)} d r .
$$

Our first condition on $c$ is $c<1 / M$. So from the convexity of $j$ we deduce that $\theta^{\prime \prime}(s)<0$ and $\theta^{\prime}(s)>0$ if $s \in\left(0, M_{0}+\delta\right]$. Moreover

$$
\theta(s) \geq e \lambda((1-c M) s) \geq e(1-c M) \lambda(s)>0 \quad \text { if } s \in\left(0, M_{0}+\delta\right] .
$$

In order to check condition (28), we observe that

$$
\theta^{\prime \prime}(s)=e \lambda^{\prime \prime}(s-c j(s))\left(1-c j^{\prime \prime}(s)\right)^{2}-e c \lambda^{\prime}(s-c j(s)) \frac{\varphi^{\prime}(s)}{\lambda(s) \lambda^{\prime}(s)} .
$$

Then, (28) is equivalent to

$$
e c \frac{\lambda(s-c j(s))}{\lambda(s)} \frac{\lambda^{\prime}(s-c j(s))}{\lambda^{\prime}(s)}\left(1-\frac{\psi(s) \sqrt{t}}{e \lambda(s-c j(s))}\right)^{2} \geq \frac{1}{2} .
$$

But the concavity of $\lambda$ implies that $\lambda^{\prime}(s-c j(s)) \geq \lambda^{\prime}(s)$. Then, from assumption (41)

$$
\left(1-\frac{\psi(s) \sqrt{t}}{e \lambda(s-c j(s))}\right) \geq\left(1-\frac{C \sqrt{t}}{e\left(1-c M_{\delta}\right)}\right) .
$$

So, condition (56) is verified if

$$
e^{2} c\left(1-c M_{\delta}\right)\left(1-\frac{2 C \sqrt{T}}{\varrho\left(1-c M_{\delta}\right)}\right) \geq \frac{1}{2}
$$

Taking $c=1 /\left(2 M_{\delta}\right),(57)$ reduces to the inequality

$$
e^{2}-4 e C \sqrt{T}-2 M_{\delta} \geq 0
$$

and, finally, the choice

$$
e=2 C \sqrt{T}+\sqrt{4 C^{2} T+2 M_{\delta}}
$$

leads to the desired estimate (42) completing the proof of Theorem 1

\section{$3.2 \quad$ Unidirectional estimates}

It is important to point out that sometimes, it is possible to get pointwise gradient estimates of unidirectional type for the solutions of the diffusion-convection Cauchy problem (35), i.e. inequalities estimates which become false if we reverse the direction of the spatial variable $x$ by making the transformation $\widetilde{x}=-x$. This is illustrated in the next result which exhibits an alternative proof to a result diven in ??.

Theorem 2. Let $u_{0} \in C^{0}(\mathbb{R})$ satisfies (36), with $M_{0}$ small enough, and let $u$ be the generalized solution to the Cauchy problem (35) corresponding to

$$
\varphi(u)=u^{m}, \psi(u)=-u^{n} \text { and } 0<n<1 \leq m .
$$


Then

$$
\left(u^{m}\right)_{x} \geq-K \frac{u^{m}}{t} \text { in } D \prime((0, T) \times \mathbb{R}),
$$

for some $K>0$ depending only on $M_{0}, m$, and $n$.

Proof. As in the proof of the previous theorem we can assume $u$ to be positive and smooth enough. Let $\lambda(r)$ be a regular function, to be chosen later, such that $\lambda(r)$ is a smooth function transforming $\left[0, w_{0}\right]$ onto $\left[0, m \ln M_{0}\right]$, for some $w_{0}>0$ and such that

$$
K=\sup _{w \in\left[0, w_{0}\right]} \lambda^{\prime}(w)>\inf _{w \in\left[0, w_{0}\right]} \lambda^{\prime}(w)=C>0 .
$$

Then, the equation

$$
m \ln u(t, x)=\lambda(w(t, x))
$$

defines a regular function $w(t, x)$ such that $0<w(t, x) \leq w_{0}$ for all $(t, x) \in[0, T] \times \mathbb{R}$. In order to prove the estimate we introduce the function

$$
z(t, x)=w_{x}(t, x) t
$$

Then, it is clear that the conclusion of the theorem will follow once we prove that $z(t, x) \geq-1$ on $[0, T] \times \mathbb{R}$, since

$$
\frac{\left(u^{m}\right)_{x}}{u^{m}}=\lambda(w)_{x}=\lambda^{\prime}(w) w_{x} \geq-\frac{K}{t} .
$$

It is not difficult to see that $z$ satisfies $\mathcal{L}(z)=0$ where

$$
\begin{aligned}
& \mathcal{L}(z)=z_{t}-m u^{m-1} z_{x x}-\frac{u^{m-1}}{t}\left[(3 m-1) \lambda^{\prime}(w)+m \frac{\lambda^{\prime \prime}(w)}{\lambda^{\prime}(w)}\right] z z_{x} \\
& -n u^{n-1} z_{x}-\frac{u^{m-1}}{t^{2}}\left[(m-1) \lambda^{\prime}(w)^{2}+m\left(\frac{\lambda^{\prime \prime}(w)}{\lambda^{\prime}(w)}\right)_{w}+(2 m-1) \lambda^{\prime \prime}(w)\right] z^{3} \\
& +\frac{n(n-1)}{m t} u^{n-1} \lambda^{\prime}(w) z^{2}-\frac{z}{t}
\end{aligned}
$$

Hence,

$$
\begin{aligned}
\mathcal{L}(-1)= & \frac{u^{m-1}}{t^{2}}\left[(m-1) \lambda^{\prime}(w)^{2}+m\left(\frac{\lambda^{\prime \prime}(w)}{\lambda^{\prime}(w)}\right)_{w}+(2 m-1) \lambda^{\prime \prime}(w)\right] \\
& -\frac{n(n-1)}{m t} u^{n-1} \lambda^{\prime}(w) z^{2}+\frac{1}{t}
\end{aligned}
$$

So, we have to choose $\lambda(r)$ such that $\mathcal{L}(-1) \leq 0$ (notice that the comparison at $t=0$ is obviously satisfied and that we can argue starting with $u_{k}$ and then passing to the limit as in the previous theorem). The above condition holds once that

$$
(m-1) \lambda^{\prime}(w)^{2}+m\left(\frac{\lambda^{\prime \prime}(w)}{\lambda^{\prime}(w)}\right)_{w}+(2 m-1) \lambda^{\prime \prime}(w) \leq 0
$$

and

$$
-\frac{n(1-n) C}{m M_{0}^{1-n}}+1 \leq 0,
$$

with $C$ given by (59). The condition (61) is satisfied by taking

$$
C \geq \frac{m M_{0}^{1-n}}{n(1-n)}
$$

To simplify the study of condition (60) we shall take $\lambda(r)$ such that $\lambda^{\prime \prime}(r) \equiv-\gamma<0$ for any $r \in\left[0, w_{0}\right]$. Then

$$
\left(\frac{\lambda^{\prime \prime}(w)}{\lambda^{\prime}(w)}\right)_{w}=\frac{-\gamma^{2}}{\lambda^{\prime}(w)^{2}}<\frac{-\gamma^{2}}{K^{2}}
$$


and (60) is satisfied once

$$
(m-1) K^{2}-m \frac{\gamma^{2}}{K^{2}}-(2 m-1) \gamma \leq 0 .
$$

Let us take $\lambda(r)=a r^{2}+b r$, with $a=(-\gamma) / 2$ and $b=K$. By taking $\gamma=K,(62)$ is transformed into

$$
(m-1) K^{2}-(2 m-1) K-m \leq 0,
$$

and so, it if fullfilled once that

$$
0<K \leq 2<\frac{(2 m-1)+\left((2 m-1)^{2}+4 m\right)^{1 / 2}}{2(m-1)} .
$$

Finally, it is a routine matter to check that, among the many possible choices, $\lambda(r)=r(2-r)$ satisfies all the requirements once we assume

$$
M_{0}^{1-n}<\min \left\{\frac{1}{2 m}, \frac{1}{\frac{m}{2}+\frac{m}{2 n(1-n)}}\right\}
$$

As we shall comment later, in Subsection 3.4, estimate (58) ceases to be true if we reverse the direction of $x$.

\subsection{Mild solutions: smoothing effects}

In the case of $u_{0} \in L^{1}(\mathbb{R})$ it is possible to show some regularizing effect for the, so called, mild solution of the Cauchy problem (35). To introduce this notion we consider the implicit timediscretization

$$
(I T D)\left\{\frac{w_{i}-w_{i-1}}{t_{i}-t_{i-1}}=\varphi\left(w_{i}\right)_{x x}+\psi\left(w_{i}\right)_{x}+f_{i} \text { in } D^{\prime}(\mathbb{R}),\right.
$$

for a given $\epsilon>0$ and a time discretization $t_{0}=0<t_{1}<\cdots<t_{n} \leq T, t_{i}-t_{i-1}<\epsilon, T-t_{n}<\epsilon$, and for given data $w_{0}, f_{i} \in L^{1}(\mathbb{R}) \cap L^{\infty}(\mathbb{R})$. We say that $u$ is a mild solution of $(35)$ if $u \in C([0,+\infty)$ : $\left.L^{1}(\mathbb{R})\right), u(0,)=.u_{0}($.$) , and, for any \epsilon>0$ there exists $\left(t_{0}, t_{1}, \cdots, t_{n}, w_{0}, w_{1}, \cdots, w_{n}\right)$ satisfying $(I T D)$ with

$$
\left\|w_{0}-u_{0}\right\|_{1} \leq \epsilon, \quad \sum_{i} \int_{t_{i-1}}^{t_{i}}\left\|f_{i}\right\|_{1} d t \leq \epsilon
$$

and such that $\left\|u(t)-w_{i}\right\|_{1} \leq \epsilon$ for any $t \in\left(t_{i-1}, t_{i}\right], i=1, \ldots, n$. The existence of a mild solution is due to Benilan and Touré [9]. Moreover, it was proven there that, under suitable additional regularity on the data $\varphi, \psi$ and $u_{0}$, the mild solution is also a weak solution.

Theorem 3. Let $\varphi$ and $\psi$ satisfy $(37)$ and let $u_{0} \in L^{1}(\mathbb{R}), u_{0} \geq 0$. Let $u \in C\left([0, T]: L^{1}(\mathbb{R})\right)$ be the mild solution to the Cauchy problem (35). Assume that

$$
R(I)=\mathbb{R},
$$

where

$$
I(u)=\operatorname{sign}(u) \int_{0}^{u}(\varphi(u)-\varphi(r)) d r .
$$

Then

$$
\|u(t, .)\|_{L^{\infty}(\mathbb{R})} \leq I^{-1}\left(\frac{\left\|u_{0}\right\|_{L^{1}(\mathbb{R})}^{3 / 2}}{4 t}\right) .
$$

Moreover, $\varphi(u(t, \cdot))_{x} \in L^{\infty}(\mathbb{R})$ and 


$$
\left\|\varphi(u(t, \cdot))_{x}\right\|_{L^{\infty}(\mathbb{R})} \leq 2\left[\frac{\varphi(m(t)) m(t)}{2}\right]^{1 / 2}+N
$$

for any $t>0$, with

$$
m(t)=I^{-1}\left(\frac{\left\|u_{0}\right\|_{L^{1}(\mathbb{R})}^{3 / 2}}{4 t}\right)
$$

and $N$ given by (46).

Proof. Using the continuity of the solution with respect to the data and the functions $\varphi$ and $\psi$ (see [8]) we may assume that $u_{0}, \varphi, \psi \in C^{\infty}(\mathbb{R})$ with $\varphi^{\prime}>0$, so that $u$ is a classical solution with $u_{x}, u_{t} \in C\left([0, T]: L^{1}(\mathbb{R})\right)$. From the definition of $I$ we deduce that $I(u)_{x}=|u| \varphi(u)_{x}$. Moreover

$$
\frac{d}{d t} \int_{\mathbb{R}} \int_{0}^{u} I(r) d r=\int_{\mathbb{R}} I(u) u_{t}=-\int_{\mathbb{R}} I(u)_{x} \varphi(u)_{x} .
$$

But, using Hölder inequality and the $L^{1}$-contraction of the associated semigroup ([9], [8]) we get that

$$
\begin{aligned}
\| I\left(u(t, .) \|_{L^{\infty}(\mathbb{R})}^{2}\right. & \leq \frac{1}{4}\left\|I(u)_{x}\right\|_{L^{1}}^{2} \leq \frac{1}{4}\left[\int_{\mathbb{R}}|u|^{1 / 2}|u|^{1 / 2} \varphi(u)_{x}\right]^{2} \leq \\
\frac{1}{4}\left\|u_{0}\right\|_{L^{1}(\mathbb{R})}\left[\int_{\mathbb{R}}|u| \varphi(u)_{x}^{2}\right] & =-\frac{1}{4}\left\|u_{0}\right\|_{L^{1}(\mathbb{R})} \int_{\mathbb{R}} I(u)_{x} \varphi(u)_{x} \\
& =-\frac{1}{4}\left\|u_{0}\right\|_{L^{1}(\mathbb{R})} \frac{d}{d t} \int_{\mathbb{R}} \int_{0}^{u} I(r) d r .
\end{aligned}
$$

On the other hand, if we define the function

$$
l(t)=\int_{\mathbb{R}}\left(\int_{0}^{u(t, x)} I(r) d r\right) d x
$$

then

$$
l(t) \leq \| I\left(u ( t , . ) \| _ { L ^ { \infty } ( \mathbb { R } ) } \| u ( t ) \| _ { L ^ { 1 } ( \mathbb { R } ) } \leq \| I \left(u(t, .)\left\|_{L^{\infty}(\mathbb{R})}\right\| u_{0} \|_{L^{1}(\mathbb{R})} .\right.\right.
$$

So, $l(t)$ satisfies the ordinary differential inequality

$$
l^{\prime}(t)+\frac{4 l(t)^{2}}{\left\|u_{0}\right\|_{L^{1}(\mathbb{R})}} \leq 0 .
$$

Dividing by $l(t)^{2}$, integrating and using that $l\left(t_{0}\right)>0$ for any $t_{0} \geq 0$ we get that

$$
l(t) \leq \frac{\left\|u_{0}\right\|_{L^{1}(\mathbb{R})}^{2}}{4 t}
$$

and finally

$$
\|I(u(t))\|_{L^{\infty}(\mathbb{R})} \leq \frac{\left\|u_{0}\right\|_{L^{1}(\mathbb{R})}^{3 / 2}}{4 t},
$$

which leads to estimate (64). The rest is an obvious application of the arguments of Corollary 4 Remark 3. It is possible to apply the general method to the study of one-side estimates on $u_{t}$. Among the many papers devoted to this task, we only mention here the presentation made in [6] and the articles [4] and [40] (many others references can be found in the surveys [31] and [41]). This allows to prove stronger differentaibilty on the solutions of (2) leading to the existence of the, so called, strong solutions satisfying that $u_{t}, \varphi(u)_{x x}$ and $\psi\left(w_{i}\right)_{x}$ are in $L^{1}((0, T) \times \mathbb{R}$ ) (see also the different approach made in [7]). 


\subsection{The case of a bounded interval}

Concerning the case in which the solution is not defined on $\mathbb{R}$ but on a bounded interval, we can get interior gradient estimates without using any concrete information on the boundary conditions. We illustrate it by considering the special case of $\psi \equiv 0$.

Proposition 1. Let $I=(a, b)$ with $-\infty<a<b<+\infty$ and $Q=(0, T) \times I$. Let $u \in L^{\infty}(Q)$ be $a$ positive solution of

$$
u_{t}=\left(u^{m}\right)_{x x} \text { in } Q \text {. }
$$

Let $\xi \in C^{2}(I), \xi>0$ on $I$ and $\xi=0$ on $\partial I$ such that

$$
\left|\xi^{\prime}(x)\right| \leq \xi(x)^{2},\left|\xi^{\prime \prime}(x)\right| \leq \xi(x)^{3} \text { for any } x \in I .
$$

Then, if $M_{0}:=\sup _{Q} u$,

a) if $0<m<1$,

$$
\left|\left(u^{m}\right)_{x}\right| \leq \frac{\xi(x) u^{m}}{\sqrt{t}}\left(\frac{\sqrt{T}}{1-m}+\sqrt{\frac{M_{0}}{2(1-m)}},\right.
$$

b) if $m=1$, for any $\varepsilon>0$,

$$
\left|\left(u^{m}\right)_{x}\right| \leq \frac{\xi(x) u}{\sqrt{t}}\left(\varepsilon+\log \frac{M_{0}}{u}\right)\left(2 \sqrt{T}\left(1+\sup \left(1, \frac{1}{\varepsilon}\right)\right)+\sqrt{\frac{2}{\varepsilon}},\right.
$$

c) if $m>1$,

$$
\left|\left(u^{m}\right)_{x}\right| \leq \frac{\xi(x) \lambda_{0}\left(\alpha u-u^{m}\right)}{\sqrt{t}}
$$

with $\alpha>\alpha_{0}:=\sup _{Q} u^{m-1}$ and $\lambda_{0}$ such that

$$
\lambda_{0} \leq \frac{2 \sqrt{T}}{(m-1)\left(\alpha-\alpha_{0}\right)}\left(m\left(\alpha-\alpha_{0}\right)+2(m-1) \alpha_{0}\right)+\sqrt{\frac{2}{(m-1)\left(\alpha-\alpha_{0}\right)}} .
$$

Proof. In the case a) we take $\theta(u)=\lambda u^{m}$. Then condition (32) is satisfied if.

$$
-\lambda^{2}(1-m)+\frac{u^{1-m}}{2}+\lambda \sqrt{t} \frac{\xi^{\prime}}{\xi^{2}}(2 m-1)+m t \frac{\xi^{\prime \prime}}{\xi^{3}} \leq 0
$$

which is implied by

$$
-\lambda^{2}(1-m)+\frac{M_{0}^{1-m}}{2}+|\lambda| \sqrt{T} \frac{\xi^{\prime}}{\xi^{2}}|2 m-1|+m T \leq 0 .
$$

This last condition holds once that

$$
|\lambda| \geq \lambda_{0}=\frac{1}{1-m}\left(\left|m-\frac{1}{2}\right| \sqrt{T}+\left(T\left|m-\frac{1}{2}\right|^{2}+(1-m)\left(2 m T+\frac{M_{0}^{1-m}}{2}\right)\right)^{1 / 2}\right) .
$$

A simple computation shows that

$$
\lambda_{0} \leq \frac{\sqrt{T}}{1-m}+\sqrt{\frac{M_{0}^{1-m}}{2(1-m)}} .
$$

Case b). We take $\theta(u)=\lambda u \log \frac{M}{u}$ with $M>M_{0}$. Then, (32) leads to

$$
-\lambda^{2} \log \frac{M}{u}+\frac{1}{2}+\frac{\xi^{\prime}}{\xi^{2}} \sqrt{t} \lambda\left(\log \frac{M}{u}-1\right)+\frac{\xi^{\prime \prime}}{\xi^{3}} t \leq 0 .
$$


Assuming $|\lambda| \geq \sqrt{T}$, then

$$
\frac{\xi^{\prime}}{\xi^{2}} \sqrt{t} \lambda \leq \lambda^{2}
$$

and the above condition becomes

$$
-\lambda^{2} \log \frac{M}{u}+\frac{1}{2}+\sqrt{T}|\lambda|\left|\log \frac{M}{u}-1\right|+2 T \leq 0 .
$$

This is satisfied once that

$$
|\lambda| \geq \lambda_{0}=\frac{1}{\log \frac{M}{u}}\left(\left|\log \frac{M}{u}-1\right| \sqrt{T}+\left(T\left(\log \frac{M}{u}-1\right)^{2}+4 \log \frac{M}{u}\left(2 T+\frac{1}{2}\right)\right)^{1 / 2}\right) .
$$

Again, a simple computation shows that

$$
\lambda_{0} \leq 2 \sqrt{T}\left(1+\frac{1}{\log \frac{M}{u}}\right)+\left(\frac{2}{\frac{1}{\log \frac{M}{u}}}\right)^{1 / 2} .
$$

Case c). We take $\theta(u)=\lambda\left(\alpha u-u^{m}\right)$ with $\alpha>\alpha_{0}:=\sup _{Q} u^{m-1}$. Then, (32) leads to

$$
-\lambda^{2}(m-1)\left(\alpha-u^{m-1}\right)+\frac{1}{2}+\lambda \sqrt{t} \frac{\xi^{\prime}}{\xi^{2}}\left(m \alpha-(2 m-1) u^{m-1}\right)+m t \frac{\xi^{\prime \prime}}{\xi^{3}} u^{m-1} \leq 0 .
$$

Assuming $|\lambda| \geq \frac{(2 m-1)}{m-1} \sqrt{T}$, then

$$
(2 m-1) \frac{\xi^{\prime}}{\xi^{2}} \sqrt{t} \lambda \leq(m-1) \lambda^{2}
$$

and the above condition becomes

$$
-\lambda^{2}(m-1)\left(\alpha-\alpha_{0}\right)+\frac{1}{2}+|\lambda| \sqrt{T}\left|m \alpha-(2 m-1) \alpha_{0}\right|+2 m T \alpha_{0} \leq 0 .
$$

This condition is satisfied if

$$
\begin{aligned}
|\lambda| \geq & \lambda_{0}=\frac{1}{(m-1)\left(\alpha-\alpha_{0}\right)}\left(\sqrt{T}\left|m \alpha-(2 m-1) \alpha_{0}\right|+\left(T\left|m \alpha-(2 m-1) \alpha_{0}\right|^{2}\right.\right. \\
& \left.\left.+4(m-1)\left(\alpha-\alpha_{0}\right)\left(\frac{1}{2}+2 m T \alpha_{0}\right)\right)^{1 / 2}\right)
\end{aligned}
$$

and we have

$$
\lambda_{0} \leq \frac{2 \sqrt{T}}{(m-1)\left(\alpha-\alpha_{0}\right)}\left(m\left(\alpha-\alpha_{0}\right)+2(m-1) \alpha_{0}\right)+\left(\frac{2}{(m-1)\left(\alpha-\alpha_{0}\right)}\right)^{1 / 2} .
$$

In the case of homogeneous Dirichlet boundary conditions and for a general maximal monotone graph $\varphi$ and $\psi \equiv 0$, it is not difficult to show that the conclusions of Corollary 4 and Proposition still hold for mild solutions. This leads to a gradient estimate which implies a regularizing effect stronger than the fact that $\varphi(u(t, \cdot))_{x} \in L^{2}(a, b)$, for any $t>0$, shown by H. Brezis in [12] for initial data $u_{0} \in H^{-1}(a, b)$. For the sake of the exposition, let us assume that

$$
D(\varphi)=R(\varphi)=\mathbb{R} .
$$

Then we have: 
Corollary 5. Let $\varphi$ be a maximal monotone graph of $\mathbb{R}^{2}$ with $0 \in \varphi(0)$ and satisfying (66). Let $u_{0} \in L^{1}(a, b), u_{0} \geq 0$. Then the mild solution $u$ of problem

$$
\begin{cases}u_{t}=w_{x x}, w \in \varphi(u) & \text { in }(0, T) \times(a, b), \\ w(t, a))=w(t, b)=0 & t \in(0, T), \\ u(0, x)=u_{0}(x) & x \in(a, b),\end{cases}
$$

satisfies

$$
\left\|w(t, \cdot)_{x}\right\|_{L^{\infty}(a, b)} \leq 2\left[\frac{\varphi^{+}(m(t)) m(t)}{2}\right]^{1 / 2}
$$

for any $t>0$, with $\varphi^{+}(r)=\max \{s \in \mathbb{R}: s \in \varphi(r)\}$ and

$$
m(t)=I^{-1}\left(\frac{\left\|u_{0}\right\|_{L^{1}(a, b)}^{3 / 2}}{4 t}\right) .
$$

\subsection{Applications to the study of the free boundaries}

We shall end the paper with several comments on the connections between pointwise gradient estimates and the study of the free boundaries defined as the boundaries of the support of the solution of the equation (2).

As it was already mentioned at the introduction, in the context of the gas flow in porous media the expression

$$
V(t, x)=-\frac{\varphi(u(x, t))_{x}+\psi(u(x, t))}{u(x, t)}
$$

represents the Eulerian description of the velocity of the "homogenized gas" at the position $x$ and the time $t$ once we identify the parabolic equation with the continuity equation for the mass conservation. In particular, if we denote by $x=\zeta(t)$ to any of those interfaces, by standard fluid mechanics arguments we deduce, at least formally, that if $\zeta(t)$ is differentiable, then the particles of the gas at the boundary of the support at time $t=0$ must remain at the boundary of the support of the gas for any $t>0$ and so they must move with velocity $V(t, \zeta(t))$. Hence if, for instance, $\zeta(t)=\sup \{x \in \mathbb{R}: u(t, x)>0\}$ then

$$
\zeta^{\prime}(t)=-\frac{\varphi\left(u(t, \zeta(t))_{x}+\psi(u(t, \zeta(t))\right.}{u(t, \zeta(t))} .
$$

The question of justifying the above formula, in a rigorous way, is not an easy task. To start with, the occurrence of the free boundaries must be correctly proved without invoking any physical argument (recall that the equation holds for an homogeneized medium). Among many papers dealing with the study of the interfaces we would start by mentioning the short paper by A.S. Kalashnikov [30] in which he shows that the solutions of the Cauchy problem (35) with $\psi \equiv 0$ and $u_{0} \geq 0$ with compact support remain with compact support for any $t>0$ (a property that, in what follows, we shall call as finite speed of propagation), provided that the pointwise gradient estimate

$$
\left|\varphi(u)_{x}\right| \leq M u
$$

holds. We recall that, by using completely different methods, it was shown that the finite speed of propagation holds if and only if

$$
\int_{0^{+}} \frac{d \varphi(s)}{s}<\infty
$$

(see [36] for the sufficient part and [27] and [37] for the necessity part). So, we conclude that estimate (69) only remains true by assuming the condition (70). Although a direct derivation of estimate (69) was already shown in [3] and [28], we mention that this can be deduced also from 
our Theorem 1 by taking $\lambda(u) \equiv u$. Notice that condition (40) coincides with (70) and that the estimate can be also proved for the class of mild solutions when $\varphi$ is a maximal monotone graph satisfying (70) since this condition make sense even if $\varphi$ is not $C^{1}$.

In the case of $\psi \neq 0$ the analysis is more sophisticated since the equation loses its invariance after changing $x$ by $-x$. The convection term privileges one direction in contrast with the other one. The finite speed of propagation (i. e. the existence of a minimal and maximal interfaces) was shown in [26] and [29] when $\varphi(u)=u^{m}$ and $\psi(u)=u^{n}$ with $m>1$ and $n \geq 1$. Nevertheless, it was shown (by means of suitable pointwise gradient estimate) in [15] that if $\varphi(u)=u^{m}$ and $\psi(u)=-u^{n}$ with $0<n<1 \leq m$ then one of the interfaces $\left(\zeta_{+}(t)=\sup \{x \in \mathbb{R}: u(t, x)>0\}\right)$ does not appear $\left(\zeta_{+}(t)=+\infty\right.$ for any $\left.t>0\right)$ although the other one $\left(\zeta_{-}(t)=\inf \{x \in \mathbb{R}: u(t, x)>0\}\right)$ exists $\left(-\infty<\zeta_{-}(t)<+\infty\right.$ for any $\left.t>0\right)$. Notice that this can be also derived from Theorem 2 since, by a comparison argument, without loss of generality, we can assume $M_{0}$ small enough and from estimate (58) we deduce that

$$
u(t, x)^{m} \geq u\left(t, x_{0}\right)^{m} e^{-\frac{K}{t}\left(x-x_{0}\right)}
$$

for any $t>0$ and $x \geq x_{0}$. Taking $x_{0}$ such that $u\left(t, x_{0}\right)>0$ we get the result. We point out that the existence of the interface $\zeta_{-}(t)$ shows the impossibility to have the reverse directional symmetric pointwise gradient estimate

$$
\left(u^{m}\right)_{x} \leq K \frac{u^{m}}{t} \text { on }(0, T) \times \mathbb{R}
$$

The occurrence of the interfaces was studied by B.H. Gilging [22], in the general case of $\varphi$ and $\psi$ as in Theorem 1 He proved that the interface $\zeta_{+}(t)$ exists if and only if there is a real number $\sigma$ such that

$$
\sigma s+\psi(s)>0 \text { for all } s \in\left(0, M_{0}\right]
$$

and

$$
\int_{0+} \frac{\varphi^{\prime}(s)}{\sigma s+\psi(s)}<\infty
$$

Notice that this proves the non existence of $\zeta_{+}(t)$ if $\varphi(u)=u^{m}$ and $\psi(u)=-u^{n}$ with $0<n<1 \leq$ $m$ and that, by changing $x$ by $-x$, it proves the existence of $\zeta_{-}(t)$. It was proved also in [22] that if $V(t, x)$ is bounded for $t=0$ then it remains bounded for any $t>0$ assumed (70) and

$$
\int_{0^{+}}\left[\frac{-\psi(s)}{s}\right] d s<\infty .
$$

Nevertheless, when $\varphi(u)=u^{m}$ and $\psi(u)=-u^{n}$ with $0<n<1 \leq m$, it was shown in [16] that the best pointwise gradient estimate has the form

$$
\left|\left(u^{m-n}\right)_{x}\right| \leq M
$$

so that estimate (69) fails. Estimate (71) was later extended in [22] to more general $\varphi$ and $\psi$ but satisfying additional conditions which are satisfied when $\varphi(u)=u^{m}, \psi(u)=-u^{n}$ and $0<n<1 \leq m$ (see also the work [25] for the study of the dependence of the Bernstein estimates on epsilon if one puts such a parameter in front of the diffusion term). We mention that, as kindly indicated in [20] and [22], the boundedness of $V$ and the generalization of the estimate (71) were obtained by applying the general idea of the method presented in this paper (a preliminar version of it circulate, as unpublished notes, among the specialists in the field since the early eighties).

The justification of the differential equation was carried out, when $\varphi(u)=u^{m}$ and $\psi(u)=-u^{n}$ in [38] and [17] for the case $0<n<1 \leq m$ (see also the formal asymptotic analysis made in [32]). It was shown $([17])$ that in ausence of waiting time phenomena $([2],[1]) \zeta_{-} \in C([0, T]) \cap \operatorname{Lip}(0, T)$,

$$
\lim _{x \rightarrow \zeta_{-}(t)-0} \frac{\varphi\left(u(t, x)_{x}\right.}{\psi(u(t, \zeta(t))}=-1
$$


and that formula (68) holds in the sense that

$$
\begin{aligned}
\zeta^{\prime}(t) & =-\lim _{x \rightarrow \zeta_{-}(t)-0} \frac{\varphi\left(u(t, x)_{x}+\psi(u(t, x)\right.}{u(t, x)} \\
& =-\lim _{x \rightarrow \zeta_{-}(t)-0}\left[\frac{\psi(u(t, x)}{u(t, x)}\right]\left[\frac{\varphi\left(u(t, x)_{x}\right.}{\psi(u(t, x)}+1\right]
\end{aligned}
$$

for a.e. $t \in[0, T]$. It is easy to see that applying (formally) the l'Hopital rule to the right-hand side of this interface equation we get a second-order equation. The interface equations of this type can also be derived by means of a special technique based on local comparison of the space profile of a solution with a set of the space profiles of a complete family of explicit solutions to the same equation, say, the travelling wave solutions: see [19], Section 5.

$C^{1+\alpha}$ and further regularity of the interface was obtained in [39] (see also [35]) under additional assumptions on the initial datum.

\section{References}

\section{References}

[1] Alvarez, L. and Díaz, J.I., Sufficient and necessary initial mass conditions for the existence of a waiting time in nonlinear diffusion-convection processes, Journal of Mathematical Analysis and Applications, 155, 1991, 378-392.

[2] Alvarez, L., Díaz, J.I. and Kersner, R., On the initial growing of the interfaces in filtration processes, in Nonlinear Diffusion Equations and Their Equilibrium States I., W.M.Ni, L.A.Peletier and J.Serrin eds.), Springer-Verlag, New York, 1988, 1-20.

[3] Aronson, D.G., Regularity properties of flows through porous media, SIAM J. Appl. Math. 17, 1969, 461-467.

[4] Aronson, D.G. and Benilan, Ph., Régularité des solutions de l'equation des mileux poroeux dans $\mathbb{R}^{N}$, C.R. Acad. Sc. Paris, 288, 1979, 103-105.

[5] Benilan, Ph., Opérateurs différentiales linéaires, in Analyse mathématique et calcul numérique pour les sciences et les techniques, R. Dautray and J.-L. Lions eds., Masson, Paris, 1984, 947-1085.

[6] Benilan, Ph., Evolution Equations and Acretive Operators, Lecture Notes (taken by S. Lenhart), Univ. of Kentucky, 1981.

[7] Benilan, Ph. and Gariepy, R., Strong solutions in $L^{1}$ of degenerate parabolic equations, Journal of Differential Equations, 119, 1995, 473-502.

[8] Benilan, Ph. and Touré, H., Sur l'équation génerale $u_{t}=\varphi(., u)_{x x}-\psi(u)_{x}+v$, C.R. Acad.Sc. Paris, 299, 1984, 919-922.

[9] Benilan, $\mathrm{Ph}$. and Touré, H., Sur l'équation génerale $u_{t}=a\left(., u, \varphi(., u)_{x}\right)_{x}+v$ dans $L^{1}$ : II- Le problème d'évolution, Ann. Inst. Henri Poincaré: Analyse non linéaire, 12, 1995, 727-761.

[10] Bernstein, S.N., Collected works, Vol III: Differential equations, Calculus of Variations and Geometry (1903-1947), Izdat. Akad.Nauk. SSSR, Moscow, 1960, (in russian).

[11] Berryman, J. G. and Holland, C. J., Stability of the separable solution for fast diffusion, Arch. Rational Mech. Anal., 74, 1980, 379-388. 
[12] Brezis, H., Monotonicity Methods in Hilbert Spaces and Some Applications to Nonlinear Partial Differential Equations, in Contributions to Nonlinear Functional Analysis, E. Zarantonello, ed., Academic Press, New York, 1971, 101-156.

[13] Carrillo,.J., Entropy solutions for nonlinear degenerate problems, Arch. Ration. Mech. Anal., 147, 1999, 269-361.

[14] Díaz, J.I., Two Problems in Homogenization of Porous Media, Extracta Mathematica, 14, 1999 141-155.

[15] Díaz, J.I. and Kersner, R., Non existence d'une des frontières libres dans une équation dégénérée en theorie de la filtration, C.R. Acad. Sc. París, 296, 1983, 505-508.

[16] Díaz, J.I. and Kersner, R., On a nonlinear degenerate parabolic equation in filtration or evaporation through a porous medium, Journal of Differential Equations, 69, 1987, 368-403.

[17] Díaz, J.I. and Shmarev, S.I., On the behaviour of the interface in nonlinear processes with convection dominating diffusion via Lagrangian coordinates, Advances in Mathematical Sciences and Applications, 1,1992, 19-45. Corrections 2, 1993, 503-506.

[18] Gagneux, G. and Madaune-Tort, M., Analyse mathematique de modeles non lineaires de l'ingenierie petroliere. (Mathematical analysis of nonlinear models of petrol engineering)., Mathematiques \& Applications, 22, Springer-Verlag, Paris, 1995.

[19] Galaktionov, V.A., Shmarev, S.I. and Vazquez, J.L., Second-order interface equations for nonlinear diffusion with very strong absorption, Commun. Contemp. Math., 1, 1999 , 51-64.

[20] Gilding, B.H., Hölder continuity of solutions of parabolic equations, J. London Math. Soc., 13, 1976, 103-106.

[21] Gilding, B.H., Properties of solutions of an equation in the theory of infiltration, Arch. Ration. Mech. Anal., 65, 1977, 203-225.

[22] Gilding, B.H., The Ocurrence of Interfaces in Nonlinear Diffusion-Advection Processes, Arch. Ration. Mech. Anal., 100, 1988, 243-263.

[23] Gilding, B.H., Improved Theory for a Nonlinear Degenerate Parabolic Equation, Annali Scu. Norm. Sup. Pisa, Serie IV, 14, 1989, 165-224.

[24] Gilding, B.H and Goncerzewicz, J., Localization of solutions of exterior domain problems for the porous media equation with radial symmetry, SIAM, J. Math. Anal. 31, 2000, 862-893.

[25] Gilding, B.H., Natalini,R. and Tesei, A., How parabolic free boundaries approximate hyperbolic fronts, Trans. Amer. Math. Soc.352, 2000, 1797-1824.

[26] Gilding, B.H. and Peletier, L.A., The Cauchy problem for an equation in the theory of infiltration, Arch. Ration. Mech. Anal., 61, 1976, 127-140.

[27] Kalasnikov, A. S., Equations of the type of a nonstationary filtration with infinite rate of propagation of perturbations, Vestnik Moskov. Univ. Ser. I Mat. Meh., 27, 1972, 45-49.

[28] Kalashnikov, A. S., On the differential properties of generalized solutions of equations of the nonsteady-state filtration type, Vestnik Moskow Univ. Ser. I Mat. Fiz., 29, 1974, 62-68.

[29] Kalashnikov, A. S., The nature of the propagation of perturbations in processes that can be decribed by quasilinear degenerate parabolic equations , Trudy Sem. Petrovsk., 1,1975, 135144 , (in russian). 
[30] Kalashnikov, A. S., On the concept of finite rate of propagation of perturbations, Uspekhi Mat. Nauk, 34, 1979, 199-200.

[31] Kalashnikov, A. S., Some problems of the qualitative theory of second-order nonlinear degenerate parabolic equations, Uspekhi Mat. Nauk, 42, 1987,135-176.

[32] Kamin, S. and Rosenau, Ph., Thermal waves in an absorbing and convective medium, Physica $8 D, 1983,717-737$.

[33] Kruzhkov, S.N., Nonlinear parabolic equations with two independent variables, Trudi Mosk. Matem. ob. (Proceedings of Moscow Math. Society), 6., 1967, 329-346.

[34] Ladyzhenskaja, O. A., Solonnikov, V. A., and Ural'tseva, N. N., Linear and quasilinear equations of parabolic type, American Mathematical Society, Providence, R.I., 1967.

[35] Meirmanov, A.M., Pukhnachov, V.V. and Shmarev, S.I., Evolution Equations and Lagrangian Coordinates, Walter de Gruyter, Berlin, 1997.

[36] Oleinik, O.A.,Kalashnikov, A.S. and Chzhou Y.-L., The Cauchy problem and boundaryproblems for equations of the type of non-stationary filtration, Izv. Akad. Nauk SSR, Ser. Mat., 22, 1958, 667-704 (in russian).

[37] Peletier, L A., A necessary and sufficient condition for the existence of an interface in flows through porous media, Arch. Ration. Mech. Anal., 56, 1974, 183-190.

[38] Shmarev S. I., Instantaneous appearance of singularities of a solution of a degenerate parabolic equation, Sib. Math. J., 31, 1990, 671-682.

[39] Shmarev, S.I., Global $C^{1+\alpha}$ regularity of interface for $u_{t}=\left(u^{m}\right)_{x x}+\left(u^{\lambda}\right)_{x}$, Advances in Mathematical Sciences and Applications, 5,1995, 1-29.

[40] Simondon, F., Strong solutions for $u_{t}=(\varphi(u))_{x x}-f(t)(\psi(u))_{x}$, Comm.in PDE, 13, 1988, 1337-1354.

[41] Vazquez, J. L., An introduction to the mathematical theory of the porousm medium equation, in Shape Optimization and Free Boundaries, M. Delfour, ed., Kluwer Acad. Publ., Dordrecht, Netherlands, 1992, 347-389. 